\title{
Optimisation De La Production De Pepiniere \\ De Tomate Sous Abri A Abidjan Sud De La Côte d'Ivoire
}

\author{
Ouattara Amidou \\ Département des Sciences du Sol, UFR des Sciences de la terre et des \\ Ressources minières, Université Félix Houphouêt-Boigny, \\ Abidjan, Côte d'Ivoire \\ Nganzoua Kouamé Réné \\ Université Jean Lorougnon Guédé Daloa, Unité de Formation et de \\ Recherche en Agroforesterie, département de Pédologie, \\ Daloa, Côte d'Ivoire \\ Adechina Olayossimi, Docteurs en sciences du sol \\ Département des Sciences du Sol, UFR des Sciences de la terre et des \\ Ressources minières, Université Félix Houphouêt-Boigny, \\ Abidjan, Côte d'Ivoire
}

Doi: 10.19044/esj.2018.v14n24p397 URL:http://dx.doi.org/10.19044/esj.2018.v14n24p397

\begin{abstract}
This study was focused in Abidjan district, located in Laguna region of Côte d'Ivoire. The aim was to determine an innovative method of growing tomato (Lycopersicum esculentum Mill) plant nursery that involves creating optimized aerial and root zone environments. This research was carried out during two successive seasons : 2015 wet season (may to august) and 2016 dry season (january to february). The tomato variety was hybrid Lindo. The plant germination, survey characters and plant vigor at each growing season as influenced by the controlled environment agriculture were identified using a completely randomized designs with four replications. The results showed a significant variability in plant germination, height, and growth vigor. Tomato plant nursery as influenced by controlled environment agriculture create optimized aerial and root zone environments, focusing on growth benefits and limited environmental impact.
\end{abstract}

Keywords: Plant nursery - Tomato - Abidjan - controlled environment

\section{Resume}

Une étude de production d'une pépinière de tomate (Lycopersicum esculentum Mill) a été conduite courant 2015 en saison pluvieuse (mai à août) 
et en 2016, en saison sèche (janvier à février) en vue d'une culture de tomate dans le périmètre maraicher de Marcory (District d'Abidjan). Elle a porté sur la variété hybride de tomate Lindo. L'objectif de l'étude est d'évaluer les conditions optima de réussite d'une pépinière. L'essai a été conduit dans un dispositif complètement randomisé avec un matériel spécifique dédié. Le taux de germination, le taux de levé, le taux de reprise au repiquage à chaque saison de culture sont les paramètres mesurés pour évaluer les performances de la pépinière. Il ressort de cette étude que la pépinière en milieu maîtrisé améliore les divers taux évalués et permet l'obtention de plants vigoureux et sains. Cela permet d'entrevoir une plantation homogène au champ.

Mots clefs : Pépinière - Tomate - Marcory - District d'Abidjan- Milieu maîtrisé

\section{Introduction}

La tomate est le légume le plus important pour l'alimentation humaine, et représente 17 p.c. de la production légumière mondiale (FAOSTAT, 2009). Cultivée essentiellement dans le maraîchage, la production de la tomate reste fortement tributaire des questions foncières en Afrique, face à une demande sans cesse croissante (Fall et Gueye 2003 ; Enda Sahel, 2003). Selon la FAO (2018), sa production reste également tributaire de la qualité de la pépinière, car en horticulture en général et en culture de tomate en particulier, la pépinière revêt une importance capitale. C'est la première étape qui conditionne la réussite de la culture. L'objectif du semis en pépinière est double : obtenir des plants vigoureux et sains. Cependant les maraichéculteurs, peinent très souvent à concevoir un semi de précision.

Vendus dans le commerce, les grains semenciers de la tomate subissent une sélection et un test de germination avant leur conditionnement et leur mise sur le marché. Le coût élevé de ces semences améliorées impose de ce fait des semis de précision. Selon George (2 009) pour réussir la pépinière, il est nécessaire de réaliser une petite serre (avec des matériaux de récupération) laissant passer la lumière et dotée d'un couvercle mobile pour faciliter l'arrosage quotidien.

La pépinière étant le lieu d'élevage de plants destinés au repiquage au champ, la vitalité ou qualité de celle-ci est un gage du succès au champ de la culture envisagée (Djiri, 2002).

Cette situation a conduit à la professionnalisation du métier de producteur de plants.

Cette étude applicative a pour objectif de répertorier les paramètres d'optimisation d'une pépinière de tomate produite sous abri. 
En effet, bien que la culture de la tomate puisse se faire directement au champ, le traitement des jeunes plants (arrosage, traitement phytosanitaire), nécessite la maitrise de la réalisation de la pépinière (George, 2009).

\section{Materiel et Methodes}

\subsection{Materiel}

\subsubsection{Matériel végétal : semences}

Il s'agit de semences améliorées de la variété de tomate Lindo vendu sur le marché dans des sachets de $1 \mathrm{~g} ; 5 \mathrm{~g}$ en boite de $50 \mathrm{~g}$ (figure 1). Pour la présente étude un sachet de $5 \mathrm{~g}$ contenant environ 1750 grains a été acheté. Les caractéristiques agronomiques de cette variété sont : fruit de forme carré, poids moyen du fruit $80 \mathrm{~g}$, précocité 65 jours et rendement 22 tha $^{-1}$ (Courchinoux, 2008).
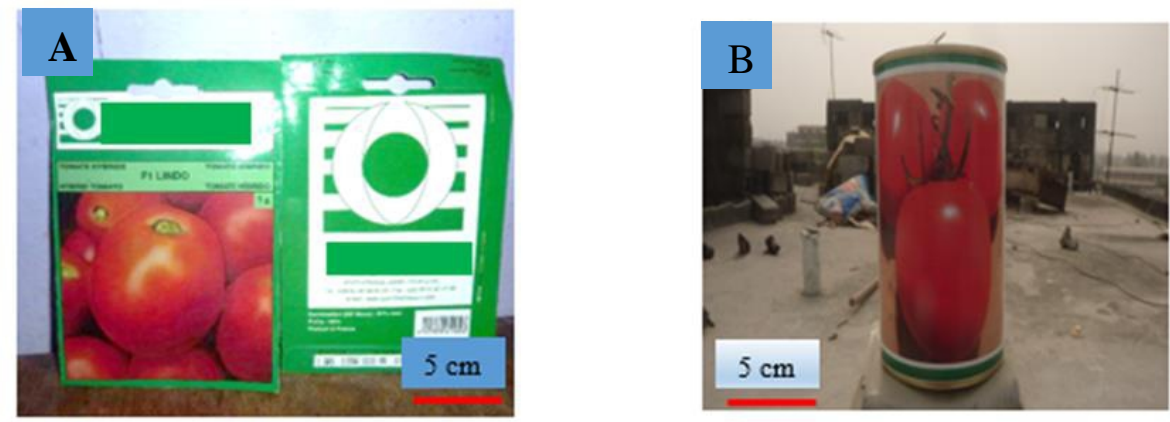

Figure 1 : Semences de tomate de la variété Lindo en sachet (gauche A) et en boite (droite B) vendues sur le marché

\subsubsection{Equipements spéciaux : substrat de culture, germoir, ombrière, pulvérisateur}

Le substrat de culture est constitué de terreau multi-usage de $20 \mathrm{~L}$, vendu sur le marché. Le terreau peut être utilisé aussi bien pour la pépinière que pour la plantation, comme fumure de fond, d'où son qualificatif de multiusage (figure $2 \mathrm{~A}$ ).

Il est composé de tourbe blonde de sphaigne, compost végétal, écorce de pin composté et d'engrais. Le $\mathrm{pH}_{\text {eau }}$ est de 6,5 avec 10 p.c. du produit brut en matière sèche et 70 p.c. de matière organique du produit sec. Ce substrat a une capacité de rétention en eau qui est de $650 \mathrm{ml} / \mathrm{L}$ et contient du NPK 1416-18.

L'ombrière est l'abri de culture de dimensions $1 \mathrm{~m}$ de long, 0,6 $\mathrm{m}$ de large et 1,20 m de hauteur conçu à l'aide de planche. Il est couvert de bâche noire et entouré de grillage. Il comporte deux niveaux dont chacun abrite trois plaques alvéolaires (figure $2 \mathrm{C}$ ).

Les germoirs sont constitués de plaques alvéolaires d'une capacité de 72 alvéoles. Ces alvéoles sont espacées de $5 \mathrm{~cm}$ avec un volume approprié 
pour l'épanouissement des plantules. Six plaques ont été payées pour les besoins de l'étude. Ce qui a donné une capacité totale de 432 alvéoles pour un nombre équivalent de futurs plants (figure 2B).
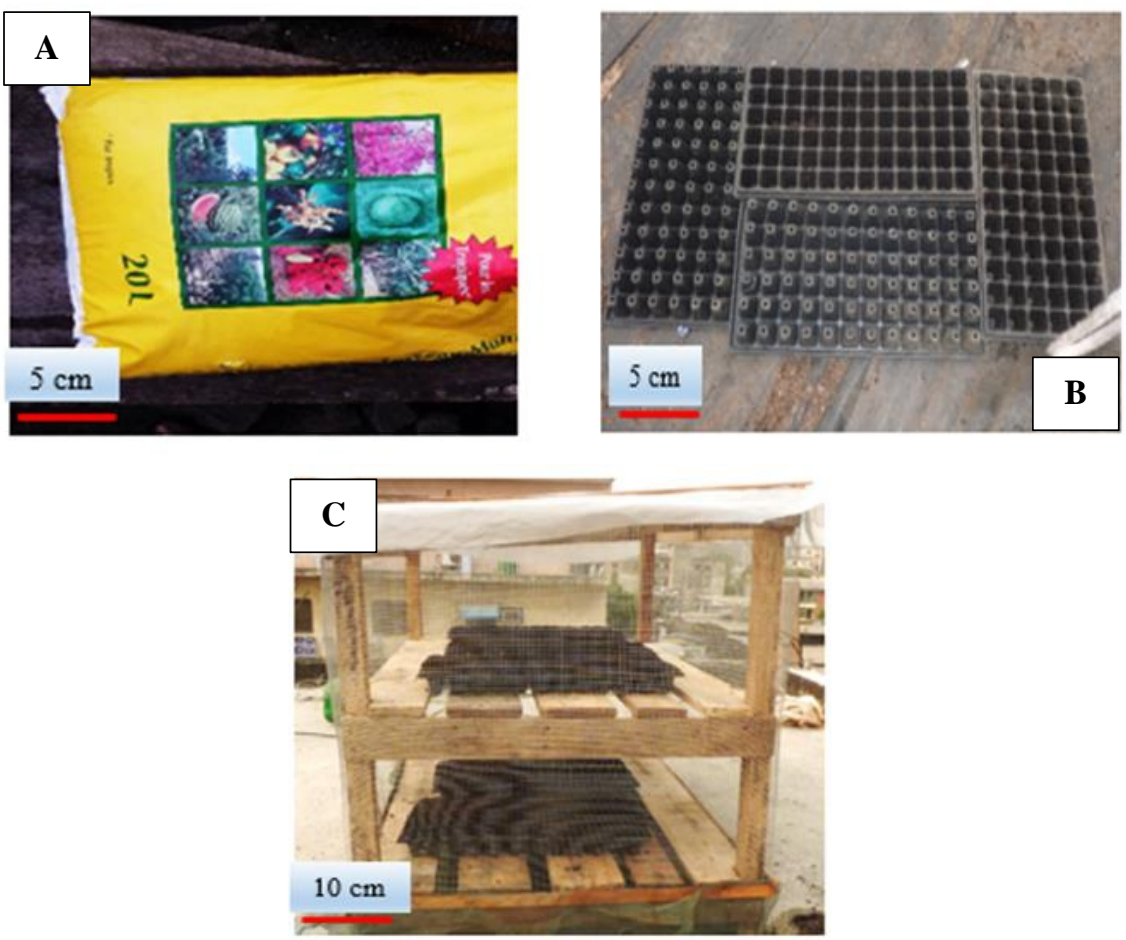

Figure 2 : Quelques éléments de l'équipement : sac de terreau (A), Plaques alvéolaires (B) et ombrière $(\mathrm{C})$

\subsubsection{Matériel d'arrosage : arrosoir, sceaux, bassines, gobelets}

C'est un contenant en plastique de $5 \mathrm{~L}$, assorti d'un entonnoir multi perforé (figure 3). Les sceaux et les bassines servent à prendre l'eau d'arrosage. Le gobelet est utilisé pour remplir l'arrosoir pour effectuer l'arrosage journalier de la pépinière à l'eau claire.

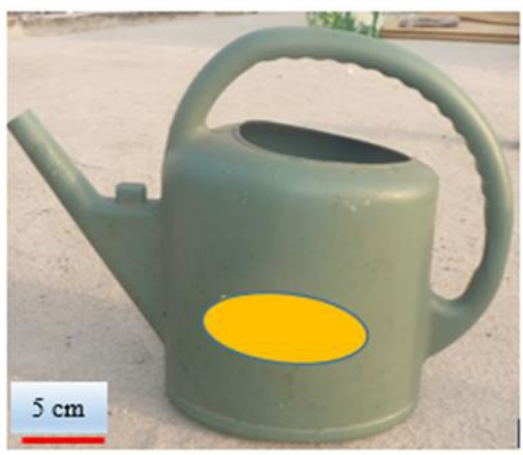

Figure 3 : Arrosoir pour les apports d'eau en pépinière de la tomate 


\subsubsection{Produit consommable : sachets plastiques, insecticides, fertilisants}

Ce sont les sachets plastiques de couleur noire ou claire, de grillage, d'engrais minéral liquide et d'insecticides.

\subsubsection{Matériels de transport : brouettes, charrettes, caisses, voitures}

Ils se composent de, palettes, de charrettes ou brouettes et de voiture.

\subsection{Methodes}

\subsubsection{Abri de culture}

A l'aide de planches, de clous, une ombrière est fabriquée par un menuisier aux dimensions et modèle prédéfinis. Elle est entreposée au lieu choisi pour réaliser la pépinière.

\subsubsection{Germoir}

Les plaques alvéolaires sont convenablement remplies avec le terreau en tassant légèrement.

\subsubsection{Semis}

Les grains sont semés par paires dans chaque alvéole en les enfouissant de façon délicate entre 2 et $3 \mathrm{~mm}$ de profondeur. Les grains ont été recouverts de fine couches de terreau ont fait l'objet d'un arrosage fin. Les germoirs sont délicatement entreposés dans l'ombrière à raison de trois par étagère. Il a été entouré de grillage et recouvert de bâche (figure 4).

\subsubsection{Paramètres mesurés}

Le taux de germination, le taux de levé et le taux de reprise, ont été évalués par traitement statistique sur l'ensemble des cycles culturaux par le logiciel XLSTAT 14.3.ou au moyen de calcul exprimé par les diverses équations qui suivront pour un seul cycle quelconque:

- Le taux de germination;

On l'estime par le nombre de grains ayant effectivement émergé du terreau au bout d'une semaine. On l'exprime par comptage et par calcul à travers l'équation suivante :

GG/NA x 100

$\mathrm{GG}=$ grains germés et $\mathrm{NA}=$ nombre d'alvéoles

- Le taux de levé ;

On l'exprime par comptage et par calcul par l'équation suivante :

PS/PG x 100

$\mathrm{PS}=$ plants ayant survécu $; \mathrm{PG}=$ plants ayant germé

- aux de reprise au repiquage ;

On l'exprime comptage et exprimé par l'équation suivante :

PSR/PR x 100 


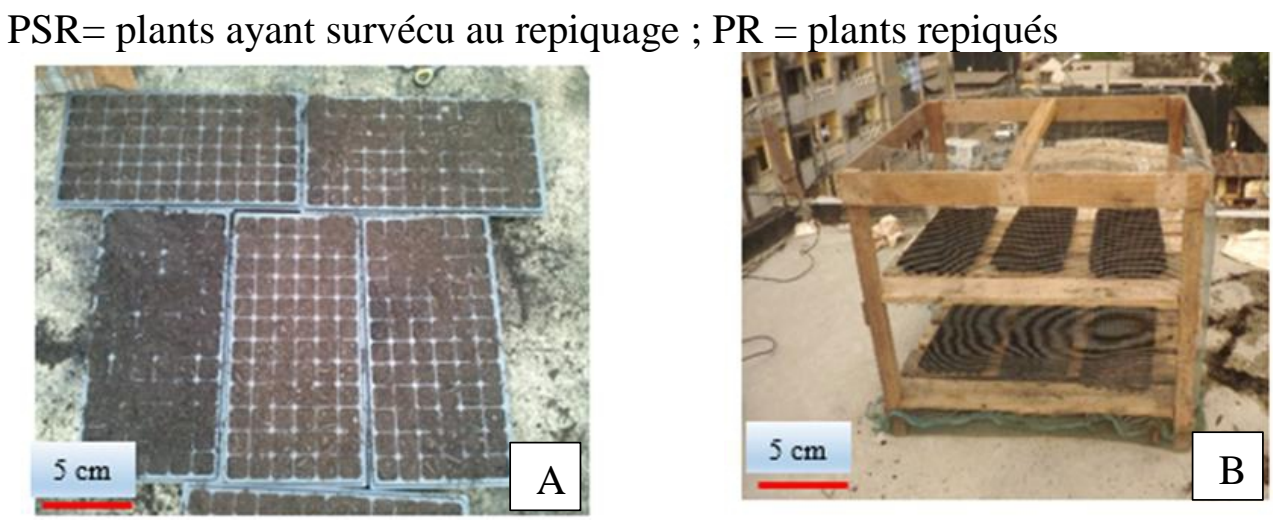

Figure 4: Plaques alvéolaires remplies au terreau (gauche A) et entreposées dans l'ombrière (droite B)

\section{Resultats et Discussion}

\subsection{Taux de germination de la variété de tomates}

La germination a été estimée au bout d'une semaine maximum après semis. Au sens du physiologiste, l'opération de germination est constatée comme atteinte lorsque l'hypocotyle perce les téguments et que la radicelle apparait. Par contre, au sens de l'agronome, la germination est accomplie après le déploiement des cotylédons et ce, une semaine environ après semis (FAO, 2018). Les analyses de variance ont révélé que, pour cette variété, les taux de germination étaient statistiquement homogènes au premier et au second, cycles de culture. Ces taux ont été respectivement de 93, 33 et 82, 33 p.c pour la variété de tomate utilisée (Tableau I). La baisse du taux enregistré au second cycle par rapport au premier cycle est lié aux mauvaises conditions climatiques (température élevée, rareté des pluies). Toutefois, sur l'ensemble des deux cycles culturaux, cette variété a enregistré en moyenne 87,83 p.c. Les pertes enregistrées sont dues à des fontes de semis. Selon Ahmed (2012), pour une bonne germination de la tomate, la réalisation de la pépinière dans des plaques alvéolées remplies de terreau de tourbe, à raison d'une graine par alvéole est à adopter.

Tableau I : Taux de germination de la variété de tomate sur les deux cycles de culture

$$
\text { Cycle } 1 \quad \text { Cycle } 2
$$

\begin{tabular}{rccc} 
Variété & mai à aout 2015 & janvier à mars 2016 & Moyenne \\
\hline LINDO & $93,33 \mathrm{a}$ & $82,33 \mathrm{a}$ & $87,83 \mathrm{a}$ \\
Probabilité $(0,05)$ & 0,005 & 0,046 & 0,001
\end{tabular}

Dans une même colonne, les valeurs suivies des mêmes lettres ne diffèrent pas statistiquement au seuil de 5 p.c. selon le test de Student Newman Keuls

Ce taux a avoisiné 95 p.c. lorsque deux grains sont semés dans chaque alvéole. Cette prédisposition est conforme aux recommandations d'Ahmed (2012) qui conseille le doublement des grains en cas de menace de déperdition. 
Toutefois dans le cas de semis double grains, un démariage s'impose, une semaine après la levée, afin de permettre un bon développement des plantules destinées au repiquage. Cette opération est destinée à supprimer les plants chétifs au profit des bons et d'aérer la pépinière (Ouattara, 2017). Selon Ahmed (2012), la confection d'une bonne ombrière influe aussi positivement sur la germination et le développement des plants en pépinière.

Il est noté que la germination survient quatre jours après semis et s'étale sur une semaine, période au terme de laquelle une évaluation du taux de germination a été effectuée. Ces observations sont conformes aux travaux de Messiaen et Lafon (1970) ; Beucher (1996) et de Soro et al., (2008) qui affirment que 4 à 5 jours suffisent aux semences pour germer en zone chaude.

\subsection{Taux de levée des variétés de tomate}

Le tableau II donne le taux de levée des plants de tomate pour la variété cultivée. Ce taux fait une estimation des plants de tomate ayant survécu à la germination au bout de trois semaines en pépinière. Les analyses de variances, ont permis de noter que la variété de tomate Lindo, a eu des taux de levée statistiquement équivalents au cours des deux cycles de culture, avec respectivement 95,67 et 84, 00 p.c. au premier et au second, cycles de culture (Tableau II). La baisse relative de ce taux au second cycle par rapport au premier cycle est liée aux conditions climatiques précédemment évoquées plus haut. Toutefois, ce taux de levée a été en moyenne 89,84 p.c. sur l'ensemble des cycles. Le taux moyen de mortalité enregistré chez la variété a été environ de 10 p.c.

Tableau II : Taux de levée des variétés de tomates sur les deux cycles de culture

\begin{tabular}{cccc}
\hline & \multicolumn{3}{c}{ Cycle 2 } \\
& Cycle 1 & janvier à & \\
Variétés & mai à aout 2015 & mars 2016 & Moyenne \\
\hline LINDO & $95,67 \mathrm{a}$ & $84,00 \mathrm{a}$ & $89,84 \mathrm{a}$ \\
Probabilité $(0,05)$ & 1 & 0,922 & 0,854 \\
\hline
\end{tabular}

Dans une même colonne, les valeurs suivies des mêmes lettres ne diffèrent pas statistiquement au seuil de 5 p.c. selon le test de Student Newman Keuls

La durée de la levée depuis la période où les plants émergent de l'alvéole à la manifestation d'un port érigé, jusqu'au repiquage dure 2 à 3 semaines après la germination (figure 5). Ces résultats sont similaires à ceux d'Akpinfa (2011), qui a trouvé dans ses études, 25 jours, le temps maximal de séjour des plants en pépinière des variétés améliorées de tomate. Un meilleur taux de levée permet d'avoir des plants de même âge pour une plantation homogène. La levée est caractérisée par une mortalité de quelques plants, avec un taux moyen de 10 p.c. Cette mortalité est attribuée aux facteurs tels que les stress hydriques, le confinement du milieu de culture, qui peuvent 
affecter négativement l'épanouissement des jeunes plantules, à cause de leur fragilité et leur sensibilité aux incidences de culture.

Ce phénomène de mortalité ou de morbidité appelé fonte de semis, est aussi attribué à un champignon straménopiles dénommé Pytium aphanidadermatium sp, appartenant à l'ordre des péronosporales, à la classe des Oomycètes et à la famille des phythiacée (Dick, 2001 et Blancard, 2009). Leur action peut se faire avant ou après la germination.
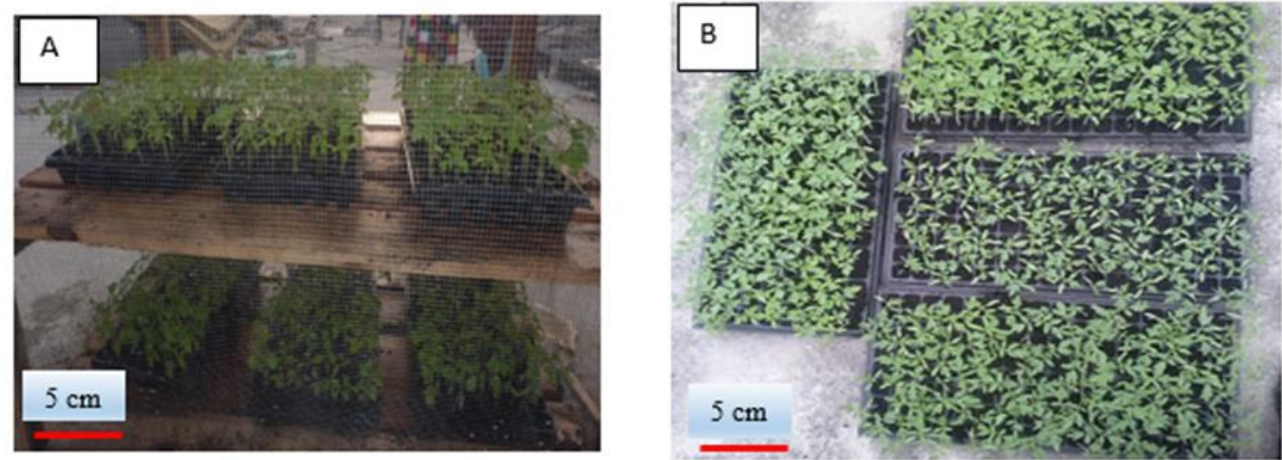

Figure 5: Quelques images des plants sous ombrière (gauche A) et hors ombrière (droite B) destinés au repiquage 3 semaines après germination

\subsection{La reprise au repiquage}

La reprise marque l'aptitude des plants à survivre au repiquage, et à poursuivre leur croissance passant par l'accomplissement des différents stades du cycle végétatif. Le repiquage se fait 3 à 4 semaines après semis (figure 6). Le temps de séjour des plants en pépinière avant leur repiquage, est compris dans l'intervalle de temps préconisé par Shankara et al. (2005) dans leur étude. En effet, ces auteurs rapportent qu'il faut un âge de 3 à 6 semaines après semis aux plantules de tomate avant de procéder à leur repiquage. Une semaine avant repiquage, les plants doivent être privés d'eau pour les habitués au stress hydrique. La reprise au repiquage a été très rapide chez la variété, et ce, quelle que soit la saison de culture, elle atteint en moyenne 80 p.c., Un fort tassement du pied de la plante au repiquage, un repiquage en temps ensoleillé, une blessure au repiquage (choc de repiquage) sont autant de facteurs pouvant causer des mortalités.

Par ailleurs, le comportement du système racinaire dans le support de culture de la variété explique la rapidité ou la difficulté à une reprise au repiquage. En effet, les tests destructifs ont montré que le système racinaire de Lindo est très voluptueux. Ainsi, Lindo colonise rapidement le sol pour assurer sa propre survie. Cette forte densité racinaire est un atout d'adaptation de la plante à son environnement de culture. Avec un développement rapide, le système racinaire de Lindo lui assure non seulement un bon ancrage, mais aussi assure les besoins nutritifs de la plante. Les études de Hanane 
(2008) Tahi (2008) sur la technique du PRD (partial rootzone drying), appliquée à la tomate, ont mis en évidence l'efficience de la faible densité racinaire quant à la résistance de la plante aux conditions de sécheresse, contrairement à une masse racinaire plus importante.
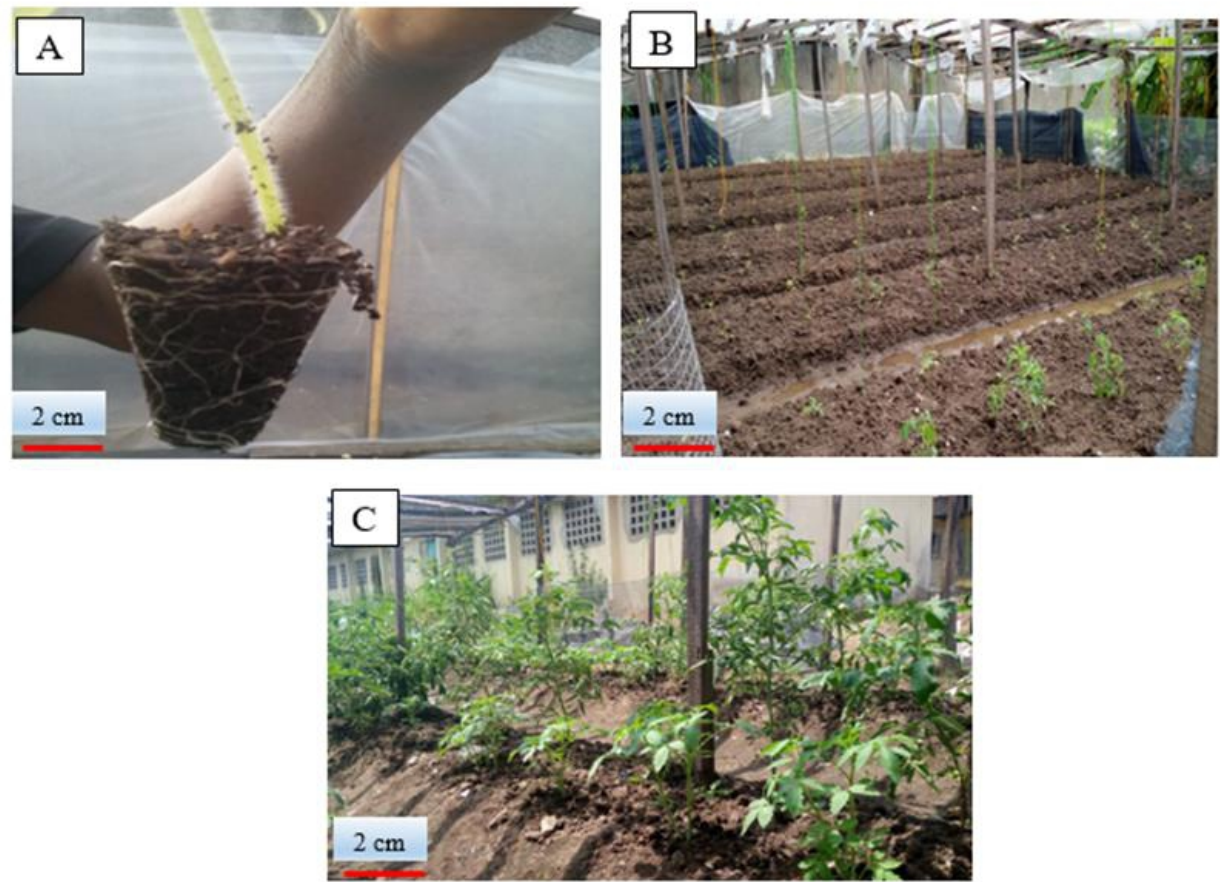

Figure 6 : Plants de tomate à différents stades du cycle. A - plant arraché de l'alvéole pour être repiqué au sol ; B- Repiquage des plants sur billons ; C- Plants après reprise au repiquage (30 jours après repiquage)

\section{Conclusion}

Au terme de notre étude, on note, d'une part que sous abri, on a obtenu des plants vigoureux et sains pour une plantation homogène au champ et d'autre part, un meilleur rendement des graines plantées et donc des jeunes plants à disposition et un bon taux de reprise au repiquage des plants matures. En outre, elle permet d'anticiper sur les besoins de l'agriculteur en tout temps, en ce sens qu'il n'est plus question d'attendre les conditions extérieures adaptées pour semer (température, pluie), mais on peut gagner quelques mois en obtenant des plantes déjà prêtes à être repiquées lorsque les conditions extérieures seront adéquates.

\section{References:}

1. Ahmed S. (2012). La tomate, www.googletamanager.com, consulté le 8 juillet 2016, $12 \mathrm{p}$. 
2. Akpinfa E. (2011). Technique de la culture de la tomate, fiche technique $\mathrm{N}^{\circ} 8,9 \mathrm{p}$.

3. Beucher P. (1996). Le jardin potager, Paris-France. 33 p.

4. Blancard D. (2009.) Les maladies de la Tomate ; Identifier, connaitre, maitriser $679 \mathrm{p}$.

5. Cournichoux J.P. (2008). Fiche technique de la tomate, technisem, Paris, 3 p.

6. Dick M.W. (2001). The peronosporomycetes. pp 59-72. In. Mc Laughlin D. and Mc Laughlin E. (Eds). The mycota, (7), Systématics and Evolution, part A, Springer Verlag, Berlin, 366 p.

7. Djiri D. (2002). Manuel de pépinière villageoise, Ministère de l'Environnement et de l'Eau, Bourkina Fasso, 25 p.

8. Enda Sahel Afrique de l'Ouest, GRAF (2003). Valorisation des acteurs et des stratégies face à l'avancée de la ville dans la périphérie et les quartiers populaires des villes secondaires comme Thiès, Enda Graf, p46. Bibliothèque Graf, $\mathrm{n}^{\circ} 487,11 \mathrm{p}$.

9. Fall S. et Gueye O. (2003). Le foncier le processus d'expansion des franges urbaines : les cas de Mboro et de Thiès, 22 p. Document interne Ecocité.

10. FAO STAT (2009). World tomatoes, all production by country, 1990$2005,11 \mathrm{p}$.

11. FAO (2016 et 2018). Production de plants de tomate en pépinière, www.fao.org/docrep/t0122f/t0122f06.htm, consulté en 30 juin 2018, $2 \mathrm{p}$.

12. George R.A.T. (2009). Vegetable Seed Production ( $3^{\text {eme }}$ édition), CAB International, in Projet 10.000 jardins potagers en Afrique, www.slowfoodfoundation.com, 4 p.

13. Hanane T. (2008). Efficience de l'utilisation de l'eau d'irrigation chez la tomate par la technique de PRD (Partial rootzone drying) et étude des mécanismes physiologiques et biochimiques impliqués, $185 \mathrm{p}$.

14. Messiaen C.M., et Lafon R. (1970). Les maladies des plantes maraichères, maladie de la tomate, de l'aubergine et du poivron. $2^{\mathrm{e}} \mathrm{ed}$., INRA, $568 \mathrm{p}$.

15. Ouattara A. (2017). Production de la tomate (Lycopersicum esculentum Solanacée Mill) sur les gleysols et essais de culture hors sol dans la commune de Marcory, sud de la Côte d'Ivoire, $125 \mathrm{p}$.

16. Shankara N., Van lidt de Jeude J., de Goffau M., Himi M., Van Dam B. et Florijin A. (2005). La culture de la tomate: production, transformation et commercialisation. $5^{\mathrm{eme}}(\mathrm{ed})$. Fondation agrosima et CTA, Wageningen, $66 \mathrm{p}$.

17. Soro S., Doumbouya M. et Koné D. (2008). Potentiel infectieux des sols de culture de tomate (lycopersicon esculentum Mill) sous abri et 
indice de l'âge de repiquage sur la vigueur des plants de pythium $\mathrm{sp}$. A Songon-Dabou en Côte d'Ivoire. Tropicultura, pp. 173-178.

18. Tahi H. (2008). Efficience de l'utilisation de l'eau d'irrigation chez la tomate par la technique de PRD (PARTIAL ROOTZONE DRYING) et étude des mécanismes physiologiques et biochimiques physiologiques et biochimiques impliqués, thèse de doctorat, biotechnologie végétale, $185 \mathrm{p}$. 